\title{
The Working Group on Space Sciences in Africa
}

Peter Martinez et al., South African Astronomical Observatory, P.O. Box 9, Observatory 7935, South Africa.

IAU membership is a good indicator of a nationally organized astronomical community. Although IAU membership statistics for Africa continue to be very poor, other indicators (such as publications) suggest that there are many individual scientists in Africa who are attempting research or promoting education in astronomy. The Working Group on Space Sciences in Africa seeks to support these individuals through various means. This poster provides an overview of astronomy in Africa and the activities of this Working Group.

Co-author is François R. Querci, France.

\section{Pollution-Free Road Lighting} lands

Duco A. Schreuder, Spechtlaan 303, 2261 BH Leidschendam, The Nether-

The beneficial effects of road lighting are often seen as very important. They relate to reducing road accidents and some forms of crime but also enhance the social safety of residents and pedestrians and the amenity for residents. Road traffic in developing countries is much more hazardous than in industrialized countries. Accident rates in 'low' income countries may be as much as 35 times higher than in 'high' income countries. Thus, it might be much more costeffective to light roads in the developing world than in the industrialized world. Fighting light pollution is more pressing in developing countries as most of the major high-class astronomical observatories are there. Astronomical observations are disturbed by light from outdoor lighting installations, part of which is scattered in the atmosphere to form 'sky glow'. The International Lighting Commission CIE has published a Technical Report giving general guidance for lighting designers and policy makers on the reduction of the sky glow.

Lighting improves visibility, essential for almost all human activity. However, light that hits the road contributes to visibility only if it is reflected. In poorly designed lighting equipment much of the lumen output of the lamps is sent directly upwards. This can be avoided by properly defined light fittings. The light output of fittings is determined by their optical quality and by the installation maintenance factor. Open fittings are to be preferred. If mounted horizontally, they make street lighting with the least light pollution.

\section{Zimbabwe - The Place for Astronomy at the next Total Solar Eclipse Francis Podmore, Dept. of Physics, University of Zimbabwe, Harare, Zimbabwe}

With clear skies most of the year, low levels of light and industrial pollution and location (we can see $96 \%$ of the celestial sphere) Zimbabwe is an excellent place for astronomy. For nearly 100 years a small but dedicated and talented band of amateur astronomers have been making hundreds of observations of occultations and variable stars, and contibuted $10 \%$ of the global total of reports to the International Halley Watch. The Astronomical Society of Southern Africa (Harare Centre) is 25 years old and the largest telescopes (mostly 'home-made') in the country are owned by members. Active preparations for the next two 
solar eclipses include site selection, coordination or safaris and free distribution of information packs and over 100000 eclipse viewers to all schools. If the economy doesn't collapse, good government and respect for law and order return, the planes keep flying and fuel shortages end, we look forward to welcoming hundreds of eclipse watchers to a dramatic 3 minute spectacle on 21 June 2001. 